OAK RIDGE

NATIONAL LABORATORY

MANAGED BY UT-BATTELLE

FOR THE DEPARTMENT OF ENERGY
CRADA FINAL REPORT

FOR

CRADA NUMBER ORNL00-0603

\title{
DEVELOPMENT OF \\ THE TWO-MODULATOR GENERALIZED \\ ELLIPSOMETER \\ (2-MGE) FOR \\ COMMERCIAL APPLICATION
}

\author{
G. E. Jellison, Jr. \\ Oak Ridge National Laboratory \\ C. O. Griffiths \\ Hinds Instruments Incorporated \\ Prepared by the \\ Oak Ridge National Laboratory \\ Oak Ridge, Tennessee 37831 \\ managed by \\ UT-Battelle, LLC \\ for the \\ U.S. Department of Energy \\ Under Contract DE-AC05-00OR22725

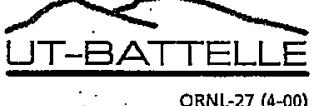




\section{DOCUMENT AVAILABILITY}

Reports produced after January 1, 1996, are generally available free via the U.S. Department of Energy (DOE) Information Bridge.

Web site http://www.osti.gov/bridge

Reports produced before January 1, 1996, may be purchased by members of the public from the following source.

National Technical Information Service

5285 Port Royal Road

Springfield, VA 22161

Telephone 703-605-6000 (1-800-553-6847)

TDD 703-487-4639

Fax 703-605-6900

E-mail info@ntis.fedworld.gov

Web site http://www.ntis.gov/support/ordernowabout.htm

Reports are available to DOE employees, DOE contractors, Energy Technology Data Exchange (ETDE) representatives, and International Nuclear information System (INIS)

representatives from the following source.

Office of Scientific and Technical Information

P.O. Box 62

Oak Ridge, TN 37831

Telephone 865-576-8401

Fax 865-576-5728

E-mail reports@adonis.osti.gov

Web site http://www.osti.gov/contact.html

This report was prepared as an account of work sponsored by an agency of the United States Government. Neither the United States Government nor any agency thereof, nor any of their employees, makes any warranty, express or implied, or assumes any legal liability or responsibility for the accuracy, completeness, or usefulness of any information, apparatus, product, or process disclosed, or represents that its use would not infringe privately owned rights. Reference herein to any specific commercial product, process, or service by trade name, trademark, manufacturer, or otherwise, does not necessarily constitute or imply its endorsement, recommendation, or favoring by the United States Government or any agency thereof. The views and opinions of authors expressed herein do not necessarily state or reflect those of the United States Government or any agency thereof. 


\title{
CRADA FINAL REPORT
}

\section{DEVELOPMENT OF THE TWO-MODULATOR GENERALIZED ELLIPSOMETER (2-MGE) FOR COMMERCIAL APPLICATION}

ORNL00-0603

\author{
G. E. Jellison, Jr. \\ Condensed Matter Sciences Division \\ Oak Ridge National Laboratory \\ and \\ C. O. Griffiths \\ Hinds Instruments, Inc. \\ Hillsboro, OR
}

\begin{abstract}
The two-modulator generalized ellipsometer (2-MGE) is an instrument that measures the change of light polarization upon interacting with a sample. The 2-MGE can operate in either reflection or transmission. In reflection, it acts as a generalized ellipsometer, measuring the standard ellipsometry parameters, as well as the cross-polarization parameters. In transmission, it measures all parameters associated with a general diattenuation and retarder.

In this CRADA, Hinds and ORNL have explored the commercial possibilities of the 2-MGE. This exploration has taken two primary paths. First, prototypes were built at both ORNL and Hinds. Second, various scientific applications were explored, including characterization of Polaroid-like materials and various materials under electric field.
\end{abstract}

\section{STATEMENT OF OBJECTIVES}

The main purpose of this CRADA was to develop the two-modulator generalized ellipsometer (2-MGE) into a commercial product. The 2-MGE is an ORNL patented technology (U. S. Patent \# 5956147 (1999)). Associated with the 2-MGE is the computer program EllipsCalc, which is used to simulate spectroscopic ellipsometry data in order to determine useful parameters, such as film thickness, surface roughness, interface thickness, and spectroscopic refractive indices, from the 2-MGE data. To this end, the CRADA had several objectives contained in two phases. 
As listed in the original CRADA (ORNL 00-0603) document dated 12/18/00, pages 13 and 14 of the Statement of Work section, they are:

Task 1. Performance Specifications.

The required performance and product cost goals for the instrument will be jointly established by Hinds and ORNL.

Status: Completed

Task 2. Order and Receive Parts.

The parts needed for the prototypes will be ordered by Hinds and ORNL.

Status: Completed

Task 3. Electrical Design.

The electronics needed for the 2MGE will be designed and built. ORNL will assist in the design, with the final circuits being designed and built by Hinds.

Status: Completed

Task 4. Mechanical Design.

The adaptors and stages required to support the 2MGE will be designed and built. ORNL will assist in the design, with the final design being done by Hinds.

Status: Completed

Task 5. Software Design.

Drivers for all components under software control will be written, as well as a graphical user interface (GUI) to allow convenient analysis, display and printing of the data. ORNL will assist in the design, with the final codework being done by Hinds.

Status: Completed

Task 6. Analysis Modules.

The ellipsometer prototype will rely upon two key analysis components within the software- one component is associated with extracting the Mueller matrix elements from the raw data collected and the other component is associated with extracting the material parameters from the Mueller matrix elements. ORNL will lead efforts to test and optimize the analysis modules.

Status: Completed

Task 7. UV and NIR Capability. 
Optical design and component selection for the 2-MGE will be investigated to determine what design criteria will be necessary to produce UV and IR systems. The objective is to identify the customer's functional requirements and to define the functional and performance specifications for UV and NIR application of 2-MGE technology.

Status: Partially Completed

Task 8. Determination of commercial feasibility for in-situ monitoring of film growth. The 2-MGE concept and its implementation in this product will be evaluated for application as an in-situ monitor of film growth. The objective is to identify the customer's functional requirements and to define the functional and performance specifications for in-situ application of 2-MGE technology.

\section{Status: Not Completed}

Task 9. Testing of prototypes.

This testing will be carried out at both Hinds and ORNL, and will involve testing the performance against the design specifications for a variety of samples. Test known samples and compare results against Rutherford backscattering (RBS) and transmission electron microscopy (TEM) techniques. If possible compare results against a rotating analyzer ellipsometer or a rotating compensator ellipsometer. The 2-MGE prototype should be tested for temperature stability as well.

Status: Completed

Task 10. First Program review.

The purpose of this review is to evaluate the results of Phase I. Key factors in the evaluation will be performance of the prototype, analysis of product cost, and confirmation of the proposed development timeline for completion of Phase II work.

Status: Completed

Addendum A 4/7/02

Statement of Work- Tasks for Phase II

Task 1. Document data analysis software.

This task will require that the software structure and modules of the data analysis software be documented. Diagram and describe code structure, define functionalities (what the code does), define all variables and constants, define/describe module interfaces. The objective of this task is to facilitate support and future modification for the data analysis software. ORNL will assist the Hinds software engineer in completing the documentation of the data analysis software.

Status: Completed 
Task 2. Document data acquisition software.

This task will require that the software structure and modules of the data acquisition software be documented. Diagram and describe code structure, define functionalities (what the code does), define all variables and constants, define/describe module interfaces. The objective of this task is to facilitate support and future modification for the data acquisition software. ORNL will assist the Hinds software engineer in completing the documentation of the data acquisition software.

Status: Completed

Task 3. Publish ellipsometry papers.

This task involves identifying novel material investigations and conducting research with the intent of publication in appropriate journals/conferences. The motivation for this task is to create a higher profile of ellipsometry using two PEMs in the scientific and industrial communities. ORNL and Hinds will cooperate in publishing papers utilizing the dual PEM ellipsometer.

Status: Completed

Task 4. Training and instruction on use and function of ellipsometer analysis methods. This task involves a transfer of knowledge from ORNL to Hinds covering the use of the analysis models and methods present in the data analysis software. The motivation for this task is that in properly assessing customer use requirements such knowledge is a necessity. In addition, support of the product will be more tractable with a firm knowledge base of ellipsometry methods internal to Hinds. The outcome of this task is a document embodying the above information

Status: Partially Completed

Task 5. Implement retardation measurement capability in the ellipsometer.

This task involves further development of the basic retardation measurement capability which is currently available in the data acquisition software. The measurement needs to cover making measurements of the magnitude of the retardation from 0 to $\pi$, the fast axis angle from 0 to $180^{\circ}$, the diattenuation from -1 to 1 , and order number $m$ for a typical retardation plate. ORNL will complete the preliminary development of the retardation measurement capability currently in the ellipsometry interface.

Status: Completed at ORNL

Task 6. Measure multilayer samples using TEM and RBS.

This task involves evaluating a set of representative samples using transmission electron microscopy and Rutherford backscattering techniques. The motivation for this task is to further clarify the accuracy of the two PEM ellipsometer relative to nonellipsometric measurement techniques. ORNL will perform testing on multilayer samples using inhouse TEM and RBS methods. 
Status: Not Completed

\section{TECHNICAL BACKGROUND}

The two-modulator generalized ellipsometer (2-MGE) is an optical instrument that is capable of simultaneously measuring 8 elements of the reduced sample Mueller matrix (out of a possible 15, where all elements are normalized to the $m_{11}$ term). If different orientations of the polarization state generator (PSG) and/or the polarization state detector (PSD) are used, then other Mueller matrix elements can be measured. Two configurations allow the determination of 13 Mueller matrix elements, while four configurations allow the determination of all 15 elements of the reduced sample Mueller matrix. $^{1}$

Figure 1 is a conceptual schematic of a general reflection or transmission ellipsometer. The incident light beam passes through the polarization state generator (PSG), which polarizes it. This beam reflects off or transmits through a sample, which changes the polarization state of the incident beam and possibly depolarizes it.

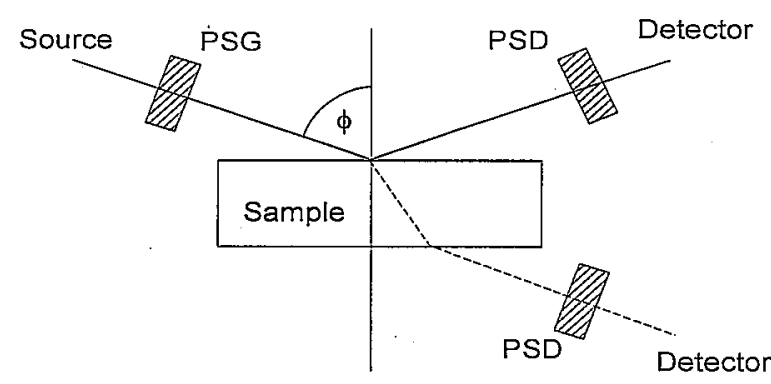

Fig. 1: A schematic of a reflection or transmission ellipsometer. The polarization state generator is labeled PSG and the polarization state detector is labeled PSD. intensity is monitored with a light detector. Usually ellipsometry measurements are performed with a large angle of incidence $\phi\left(\sim 65^{\circ}\right)$.

The details of the 2-MGE are discussed in Refs. 1. The instrument consists of two polarizer-photoelastic modulator (PEM) pairs, one acting as the PSG (0), the other as the PSD (1). The PSG polarizer-PEM pair dynamically elliptically polarizes the light beam at its resonant frequency $\omega_{0}=50.2$ and the second PEM-polarizer pair dynamically repolarizes the light beam from the sample at a different resonant frequency $\omega_{1}=60.2 \mathrm{kHz}$. The resulting intensity waveform at the detector is of the form

$$
\begin{aligned}
& I(\mathrm{t})=I_{d c}+I_{X 0} X_{0}+I_{Y O} Y_{0}+I_{X I} X_{I}+I_{Y I} Y_{1}+I_{X O X I} X_{0} X_{1}+I_{X O Y I} X_{0} Y_{1}+I_{Y O X I} Y_{0} X_{I} \\
& +I_{Y O Y 1} Y_{O} Y_{1},
\end{aligned}
$$

where 


$$
\begin{aligned}
& X_{i}=\sin \left(A_{i} \sin \left(\omega_{i} t\right)\right), \\
& Y_{i}=\cos \left(A_{i} \sin \left(\omega_{i} t\right)\right), \quad i=0,1
\end{aligned}
$$

The Bessel angles $A_{i}$ are proportional to the modulating amplitude of each modulator. The time-dependent intensity is extremely complicated, having as many as 30 Fourier components greater than $10^{-3}$ times the average light intensity $I_{d c}$. However, the individual Fourier components are naturally attenuated at higher frequencies, having no significant Fourier component for frequencies greater than $400 \mathrm{kHz}$. Using a Fourier-like analysis, the eight coefficients $I_{X O}, I_{Y O}, I_{X I}, I_{Y 1}, I_{X O X I}, I_{X O Y 1}, I_{Y O X I}$, and $I_{Y O Y I}$ are measured relative to $I_{d c}$ that serves as a normalization factor. If the instrument is properly calibrated and aligned, the 8 parameters are independent and are equal to 8 different elements of the normalized Mueller matrix (possibly times a sign factor) associated with light reflection from the sample surface. For azimuthal orientations of the PSG and the PSD that are $\left(0^{\circ}\right.$, $\left.\pm 45^{\circ}\right),\left(90^{\circ}, \pm 45^{\circ}\right)\left( \pm 45^{\circ}, 0^{\circ}\right)$, or $\left( \pm 45^{\circ}, 90^{\circ}\right)$, then either the upper-right or the lower-left block of the sample Mueller matrix is completely measured. If the sample is nondepolarizing, then light reflecting from the sample can be described using the sample Mueller-Jones matrix and all 6 elements of the normalized Jones matrix $\mathbf{J}$ can be determined (see Refs. 1 for details).

The 2-MGE is spectroscopic in that the light source is a white-light Xe arc lamp and a monochromator is placed in front of the photomultiplier tube detector. Our system works between 220 and $880 \mathrm{~nm}$ (5.64 to $1.41 \mathrm{eV})$. As with all ellipsometers, accurate measurements require accurate calibrations. The amplitude of the PEM modulation must be determined as a function of wavelength; this calibration. usually requires that the amplitude of the modulation be set at each wavelength such that the Bessel angles $A_{i}$ are equal to a known value (usually 2.405). In addition, the static strain of each modulator must be measured as a function of wavelength, and the azimuthal angle of the polarizer with respect to each photoelastic modulator must be set to $\pm 45^{\circ}$. For cases where lenses are used to focus the light spot, the strain-induced retardation of the lenses must be measured and included in the data reduction; this is particularly easy with the 2-MGE.

Previously, the main application of the 2-MGE has been in reflection, where the instrument behaves as a generalized spectroscopic ellipsometer. ${ }^{1-7}$ In this configuration, the instrument measures the quantities $\mathrm{N}, \mathrm{S}$, and $\mathrm{C}$ of isotropic samples, thereby allowing for the unambiguous determination of $\psi$ and $\Delta$, as well as the degree of polarization. The $2-\mathrm{MGE}$ is also capable of determining cross-polarization terms associated with anisotropic samples (such as uniaxial crystals or diffraction gratings) using the measurements from a single configuration. If the optic axis of a uniaxial crystal is significantly off-normal, then it is possible to determine the spectroscopic refractive index and extinction coefficient for light polarized parallel and perpendicular to the optic axis with a single spectroscopic measurement.

Clearly, the same instrument can be operated in transmission mode. In this case, a single measurement of the 2-MGE still determines 8 Mueller matrix elements, but the interpretation of the measured quantities is considerably different. It is possible to 
completely characterize a linear diattenuator and retarder using a single measurement, where the diattenuation $\mathrm{N}$, the retardation $\delta$, the direction of the principal axis $\phi$, the polarization factor $\beta$ and the circular diattenuation are all determined. Furthermore, $\delta$, and $\phi$ can often be determined to \pm 0.001 radian and $N$ to \pm 0.001 , regardless of their values.

The transmission ellipsometry measurements made with the 2-MGE can be performed in two different modes. ${ }^{8}$ The first mode of operation utilizes a broadband light source to illuminate the whole sample. The transmitted beam is then imaged onto a spectrometer, which allows the measurements to be performed as a function of wavelength. The second mode of operation is performed at a single wavelength, but areas of the sample are sequentially imaged onto the end of a fiber connected to a photomultiplier tube. The imaging optic is motion-controlled, resulting in a "mapping" of the sample. In both cases, the parameters $\mathrm{N}, \delta, \phi, \beta$ and the circular diattenuation are measured either as a function of wavelength or as a function of spatial position.

\section{EXAMPLES OF THE SCIENTIFIC WORK PERFORMED DURING THE CRADA}

The scientific work performed during this CRADA took many forms. Some of the work involved separate evaluations of standard samples both at ORNL and. Hinds in reflection (that is, standard ellipsometry). Such samples included silicon, silicon dioxide, thin films of silicon dioxide grown on silicon, etc. In all cases, the final results indicated that both systems were operating similarly.

To explore additional applications of the 2-MGE, the ORNL system was set up in the transmission configuration, where it could be operated either spectroscopically or as an $\mathrm{x}$ $y$ mapping system. There were several samples examined using this system, two of which are described below:

\section{A. Polaroid}

Figure 2 shows the diattenuation $(N), S$, and $C$ of a Polaroid polarizing film as a function of wavelength (using the configuration shown in Fig. 1a), through a $2.5 \mathrm{~mm}$ diameter aperture. According to the data in Fig. 2, the Polaroid film is a very good polarizer from $\sim 520-720 \mathrm{~nm}$ (where $N=-1, S=C=0$ ), but deviates significantly from ideal both in the blue and the red parts of the spectrum. Although the diattenuation remains very close to -1 in the blue and the ultraviolet, there is a significant amount of retardation (as indicated by the increased magnitude of $S$ and $C$ ), and there is some depolarization below $\sim 370 \mathrm{~nm}$ (not shown). Very little light below $325 \mathrm{~nm}$ passes through the Polaroid material. The effects in the red part of the spectrum are even more dramatic: the diattenuation rapidly drops off from -1 , while $S$ and $C$ become more important. While the Polaroid is a very good polarizer at $720 \mathrm{~nm}$, at $850 \mathrm{~nm}$, the Polaroid is more of a retarder than a polarizer! 
Figure 3 shows the X-Y scan of the Polaroid taken at $830 \mathrm{~nm}$ (taken using the configuration shown in Fig. 1b). As can be seen from Fig. 2, the $N, S$, and $C$ parameters are all significantly different from zero at this wavelength, meaning that the Polaroid is a complicated linear diattenuator and retarder at this wavelength. The most interesting part of this figure is that the retardation of the Polaroid varies considerably over the aperture, and the point-by-point changes in retardation mirror similar changes in the diattenuation. However, similar changes are NOT observed in the direction of polarization/fast axis, which remains constant at $176.1^{\circ}$. However, the direction of polarization/fast axis map does indicate a defect near the edge, at $\sim-11$ o'clock. This defect can be seen in the intensity plot, but not in either the retardation or the diattenuation maps. Several defects can be observed in both the retardation and diattenuation maps that are not reflected in either the intensity or the fast axis maps.

A similar X-Y scan was taken of the Polaroid at $633 \mathrm{~nm}$, but these results were unremarkable in their consistency, where the only defect to be seen, other than the defects in the intensity, was the defect in the direction of the fast axis/polarization map near the edge at $\sim 110^{\prime}$ 'clock. This is because the Polaroid in nearly a perfect polarizer at $633 \mathrm{~nm}$, that is, the retardation parameters $(S$ and $C$ ) are very small.

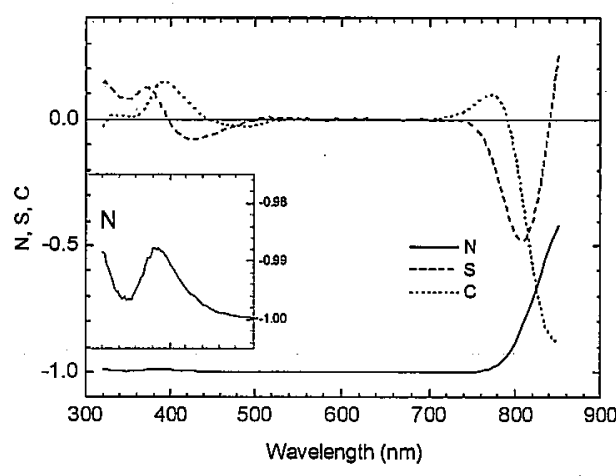

Figure 2: The diattenuation $N$, and the $S$, and $C$ parameters for a Polaroid polarizer. The inset shows a magnified plot of $\mathrm{N}$ in the ultraviolet and blue parts of the spectrum.

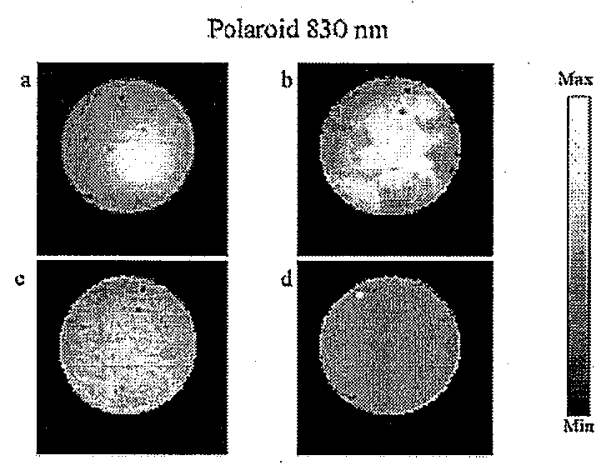

Figure 3: $x-y$ scanning maps of a) the intensity of the light beam through the sample, b) the retardation, $c$ ) the diattenuation, and d) the principal axis direction. The maximum (white) and minimum (black) values are: a) Intensity $(0-1.0$, relative scale $), b)$ retardation $(2.5-3.0 \mathrm{rad}), \mathrm{c})$ diattenuation $(-0.75--0.55) . \mathrm{d})$ principal axis direction $\left(175^{\circ}-178^{\circ}\right)$

\section{B Electric field-induced birefringence in $\mathrm{LiNbO}_{3}$}

One very interesting application of the 2-MGE is the measurement of electric fieldinduced birefringence in non-centrosymmetric crystals, such as lithium niobate $\left(\mathrm{LiNbO}_{3}\right)$. For a generally applied electric field, the impermeability tensor of a trigonal, $3 \mathrm{~m}$ symmetrical, uniaxial material (such as $\mathrm{LiNbO}_{3}$ ) is given by ${ }^{9}$ 


$$
\boldsymbol{\eta}=\left(\begin{array}{ccc}
\eta_{o}+r_{13} E_{z}-r_{22} E_{y} & -r_{22} E_{x} & r_{51} E_{x} \\
-r_{22} E_{x} & \eta_{o}+r_{13} E_{z}+r_{22} E_{y} & r_{51} E_{y} \\
r_{51} E_{x} & r_{51} E_{y} & \eta_{e}+r_{33} E_{z}
\end{array}\right),
$$

where the applied electric field vector is given by $\mathbf{E}=E_{x} \mathbf{i}+E_{y} j+E_{z} k$. The matrix in $E q$. 1 is diagonalized (using perturbation theory), resulting in the eigenvalues:

$$
\begin{aligned}
& \lambda_{1}=\eta_{0}+r_{13} E_{z}+r_{22} E_{0} \\
& \lambda_{2}=\eta_{0}+r_{13} E_{z}-r_{22} E_{o} \\
& \lambda_{3}=\eta_{e}+r_{33} E_{z}
\end{aligned}
$$

where $E_{0}^{2}=E_{x}^{2}+E_{y}^{2}$.

The refractive indices are given by $n_{i}^{2}=1 / \lambda_{i}, i=1,2,3$. (For $\mathrm{LiNbO}_{3}$ at $\lambda=633 \mathrm{~nm}, \mathrm{n}_{0}=$ 2.286 and $n_{e}=2.200$; see ref. 13). The application of the field does not appreciably change the direction of the $z$-axis, but rotates the $x$-and $y$-axes (now no longer degenerate) by the angle

$$
\tan \phi=\left(\frac{E_{o}+E_{y}}{E_{x}}\right)=\cot \left(\frac{\theta}{2}\right)
$$

where $\theta=\tan ^{-1}\left(E_{x} / E_{y}\right)$. For light passing along the z-axis, the observed birefringence is $\Delta \mathrm{n}=-\mathrm{n}_{\mathrm{o}}{ }^{3} \mathrm{r}_{22} \mathrm{E}_{\mathrm{o}}$ resulting in the retardation

$$
\delta=\frac{2 \pi d \Delta n}{\lambda}=-\frac{2 \pi d n_{o}^{3} r_{22} E_{o}}{\lambda}
$$

Figure 4 shows the $x-y$ maps of several parameters of a congruently grown $\mathrm{LiNbO}_{3}$ sample (uniaxial crystal with trigonal symmetry, $3 \mathrm{~m}$ point group) under $1000 \mathrm{~V}$ bias. The crystal was oriented such that the $z$-axis is pointed along the light beam, and the voltage is applied along the $\mathrm{x}$-axis of the crystal as shown. Under zero bias, the sample is nominally isotropic (showing an average retardation of $0.008 \mathrm{rad}$ with a standard deviation of $0.005 \mathrm{rad}$ and an average error of $0.002 \mathrm{rad}$ ) and exhibits only a small amount of retardation due to strain or local variations of the c-axis of the crystal. (Recall that congruently grown $\mathrm{LiNbO}_{3}$ is not stoichiometric, so it may have regions of varying Li concentration.) The intensity map shows that the light intensity varies considerably over the $x-y$ map, but does not appreciably affect the values of the determined parameters, such as the retardation, principal axis direction, or diattenuation. However, there are two obvious defects in the crystal (indicated by the blacker spots in the intensity map) where considerably less light gets through the sample. These defects can also be seen in the retardation and the diattenuation, but the affect on the principal axis angle is the most noteworthy, where it can be seen that there is "swirl" or miss-orientation near the defect. Under the $1000 \mathrm{~V}$ bias, there is a non-zero retardation throughout the sample 


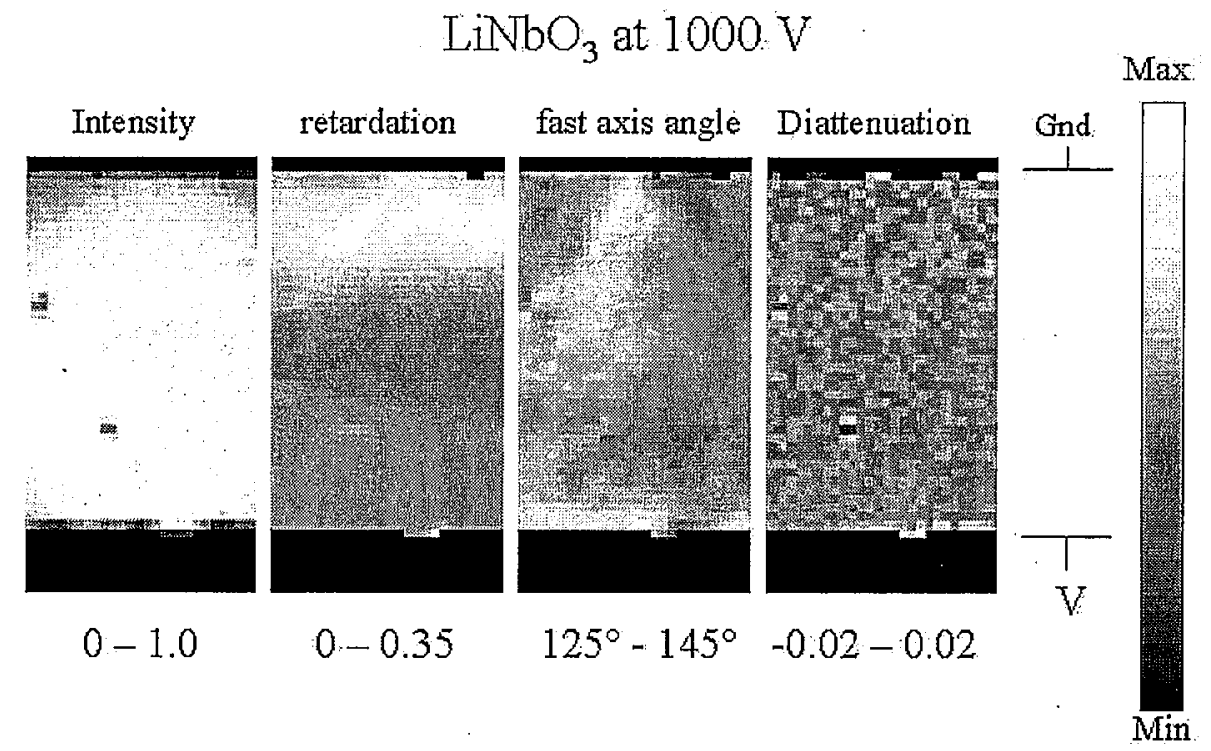

Figure 4. An $x-y$ map of the light intensity, retardation, fast axis angle, and diattenuation of a congruently grown $\mathrm{LiNbO}_{3}$ sample oriented with the $\mathrm{z}$-axis out of the page, where the voltage is applied as shown along the $\mathrm{x}$-axis of the crystal. The maximum and minimum of the black-and-white scale is given at the bottom of the figure. The pixel size is $\sim 40$ microns, and the distance between the applied voltage and ground is 1.94 $\mathrm{mm}$.

(indicating a non-zero field), but an obvious increase of the retardation near the ground contact. Moreover, there are distinct features in both the retardation and in the principal axis angle near the ground contact that make an angle $\sim 45^{\circ}$ with respect to the contact, indicating a line defect in the crystal, possibly due to twinning. of the non-stoichiometric, congruently grown, $\mathrm{LiNbO}_{3}$ material.

Interestingly, the retardation and the fast axis angle are NOT constant throughout the sample. The principal axis angle is centered at $\sim 135^{\circ}$ (as one would expect from the discussion in section 2.3 above), but with considerable variation about this value. Furthermore, the retardation can vary by as much as a factor of 2 from one point to another. The variation in retardation (see Eq. 5) can be due to either a variation of the actual electric field in the material, or due to a variation of the $r_{22}$ coefficient (from the non-stoichiometric nature of congruently grown $\mathrm{LiNbO}_{3}$ ). The variation of the principal axis angle is probably indicative that there are local variations in the crystallographic axes within the material. 


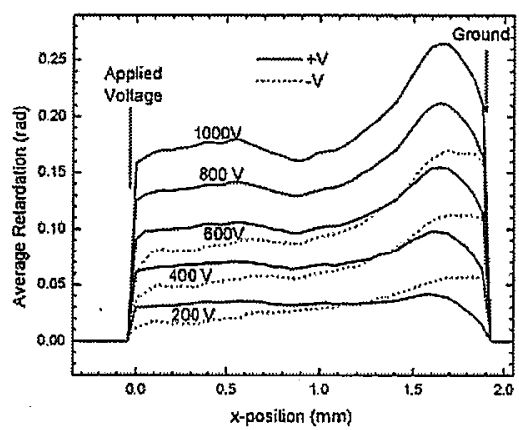

Figure 5 . The average retardation of the data shown in Fig. 4 and other data sets taken at different voltages using the congruently grown $\mathrm{LiNbO}_{3}$ sample. The $\mathrm{x}$-position is along the $\mathrm{x}$ axis of the crystal and coincides with the application direction of the voltage.

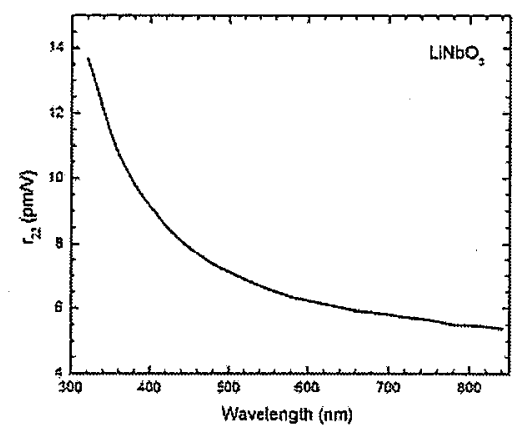

Figure 6. The average value of the Pockels coefficient $r_{22}$ as a function of wavelength.

Figure 5 shows the averaged retardation (where the averaging is done parallel to the contacts) for several different voltages. The sample was first set to the indicated voltage, and then held at that voltage for at least $10 \mathrm{~min}$ before a measurement was taken. Clearly, the shape of this curve increases in a monotonic way with increased voltage, and there is an obvious difference between positively applied voltages and negatively applied voltages. However, the increased retardance near the ground plane is clearly indicated. The averaged value the retardation over the $\mathrm{x}$-position is linear with applied voltage, and results in an averaged Pockels coefficient $\left\langle\mathrm{r}_{22}\right\rangle=6.08 \mathrm{pm} / \mathrm{V}$. (Compare to literature values ${ }^{10}$ of $3-7 \mathrm{pm} / \mathrm{V}$.)

A spectroscopic measurement of the electric field induced birefringence in congruently grown $\mathrm{LiNbO}_{3}$ was performed using the spectroscopic experimental configuration. For this experiment, all the light passing through the sample was collected onto the end of a much larger fiber ( 400 micron core), so there is an integration both over the area of the sample and over the light intensity incident upon the sample, neither one of which are constant. However, the average value of the Pockels coefficient $r_{22}$ can be determined from the retardation data (see Eq. 5), where the spectroscopic values of the ordinary refractive index $n_{0}$ was obtained from ref. 11 . The spectroscopic values of $r_{22}$ are shown in Figure 6. As can be seen, the $r_{22}$ Pockels coefficient is extremely dispersive, changing by a factor of 3 over the measured wavelength. Accurate measurements can be made down to $\sim 320 \mathrm{~nm}(\sim 3.87 \mathrm{eV})$, where the crystal became totally opaque. 


\section{INVENTIONS AND PAPERS RESULTING FROM THE CRADA}

Most of the work on this CRADA involved the building of prototypes at Hinds and at ORNL, and in exploring various applications of the 2-MGE. The 2-MGE is patented under U. S. Patent \# 5956147, held by ORNL, and licensed to Hinds Instruments, Inc. This patent includes both reflection and transmission experiments, so it covers all aspects of the experimental work performed during this CRADA. The software program "EllipsCalc" has been copyrighted by ORNL, and this too has been licensed to Hinds.

The applications of the 2-MGE were deemed to be publishable both by the management of the Condensed Matter Sciences Division and by Hinds Instruments, Inc. Below are listed the publications resulting in part from the work performed during this CRADA:

1. G. E. Jellison, Jr., C. O. Griffiths, and D. E. Holcomb, "Electric field-induced birefringence in $\mathrm{LiNbO}_{3}$ measured by generalized transmission ellipsometry," Appl. Phys. Lett. 81, 1222-1224 (2002).

2. G. E. Jellison, Jr., C. O. Griffiths, D. E. Holcomb, and C. M. Rouleau, "Transmission 2-modulator generalized ellipsometry (2-MGE) measurements," Appl. Opt. 41, 65556566 (2002).

3. G. E. Jellison, Jr. C. O. Griffiths, D. E. Holcomb, and C. M. Rouleau, "Characterization of linear diattenuator and retarders using a two-modulator generalized ellipsometer (2-MGE)," SPIE 4819 9-19 (2002).

4. G. E. Jellison, Jr., S. P. Withrow, S. Jaiswal, C. M. Rouleau, J. T. Simpson, C. W. White, C. O. Griffiths, "Spectroscopic ellipsometry studies of nanocrystalline silicon in thin-film silicon dioxide," Mat. Res. Soc. Symp. (2003) in press.

5. G. E. Jellison, Jr., D. E. Holcomb, C. O. Griffiths, M. Grozas, A. Burger, L. Li, and F. $\mathrm{Yu}$, "Internal electric field measurements in cadmium zinc telluride (CZT) using transmission two-modulator generalized ellipsometry," J. Elect. Mat (2003) in press.

\section{COMMERCIALIZATION}

Hinds and ORNL have made several contacts with third party companies that manufacture products or materials for which the 2-MGE would be a critical diagnostic. Several of the potential applications are listed below:

1. Thin-film polarizers. 2-MGE measurements were made in transmission on a series of their samples, both spectroscopically and using the $x-y$ mapping function.. The three parties are exploring collaboration possibilities.

2. Materials under electric field. Many materials are required to operate under a reasonably high electric field to perform their function. Two such materials are $\mathrm{LiNbO}_{3}$, which is used as an optical modulator, and Cadmium Zinc Telluride (CZT), which is used as a room temperature gamma detector. We are currently exploring the possibility of 
collaborating with small businesses, universities (Fisk University), and at National Labs (Oak Ridge and Brookhaven) to use the 2-MGE as a primary diagnostic for crystal growth of materials that must operate under intense electric fields.

3. Thin film diagnostics. Operating in reflection, the 2-MGE is unparalleled as a generalized ellipsometer. That is, it can measure all the standard ellipsometry parameters, as well as the cross-polarization effects. As such, it has unique opportunities for thin-film diagnostics in a variety of applications.

\section{FUTURE COLLABORATION}

Although the work of the CRADA has successfully built prototypes at ORNL and at Hinds, the work to commercialize the 2-MGE is far from complete. It is anticipated that future work will include instrument development to improve the 2-MGE, software development to improve the understanding of 2-MGE data, and the exploration of additional applications. We are presently setting up a work for others agreement between ORNL and Hinds to accomplish this.

\section{CONCLUSIONS}

The CRADA successfully built two prototypes, one at ORNL and one at Hinds Instruments. Measurements involving the exchange of samples indicated that both systems worked within specifications. A commercial beta version of the software package "EllipsCalc" has been constructed and will be expanded in the near future. Several interesting scientific studies have been performed using the 2-MGE. In transmission, the 2-MGE is capable of completely characterizing polarizers such as Polaroid, and of detecting electric field-induced birefringence in several materials.

\section{ACKNOWLDEGEMENTS}

This work was funded in part by the Department of Energy Office of Science Laboratory Technology Research Program under contract DE-AC05-00OR2275 with Oak Ridge National Laboratory, managed by UT-Battelle, LLC. 


\section{REFERENCES}

1. G. E. Jellison, Jr. and F. A. Modine, "Two Modulator generalized ellipsometry: experiment and calibration," Appl. Opt. 36, 8184-8189 (1997); "Two Modulator generalized ellipsometry: theory," Appl. Opt. 36, 8190-8198 (1997).

2. G. E. Jellison, Jr., F. A. Modine, and L. A. Boatner, "Measurement of the optical functions of uniaxial materials by two-modulator generalized ellipsometer: rutile $\left(\mathrm{TiO}_{2}\right)$, Opt. Lett. 22, 1808-1810 (1997).

3. G. E. Jellison, Jr. and L. A. Boatner, "Optical functions of uniaxial $\mathrm{ZnO}$ determined by generalized ellipsometry," Phys. Rev. B, 58, 3586-3589 (1998).

4. G. E. Jellison, Jr. J. O. Ramey, and L. A. Boatner, "Optical functions of $\mathrm{BiI}_{3}$ as measured by generalized ellipsometry," Phys. Rev. B. 59, 9718-9721 (1999).

5. G. E. Jellison, Jr., F. A. Modine, and L. A. Boatner, "Measurements of the optical functions of uniaxial crystals using 2-modulator generalized ellipsometry (2-MGE)," SPIE 3425 232-238 (1998).

6. G. E. Jellison, Jr., L. A. Boatner, and C. Chen, "Spectroscopic refractive indices of metalorthophosphates with the zircon-type structure," Opt. Materials, 15, 103-109 (2000).

7. G. E. Jellison, Jr., F. A. Modine, and C. Chen, "Calibration procedures for a twomodulator generalized ellipsometer," SPIE, 3754, 150-160 (1999).

8. G. E. Jellison, Jr., C. O. Griffiths, D. E. Holcomb, and C. M. Rouleau, "Transmission 2-modulator generalized ellipsometry (2-MGE) measurements," Appl. Opt. 41, 65556566 (2002).

9. T. A. Maldonado, Electro-optic modulators, Ch. 13 in Handbook of Optics, Vol. II (McGraw-Hill, New. York, M. Bass, ed. 1995).

10. Y. Xu, Ferroelectric Materials and their applications, (North Holland, New York, 1991).

11. E. D. Palik, Handbook of Optical Constants of Solids, (Academic Press, New York, E. D. Palik, ed. 1985), p. 698. 
C/ORNL00-0603

\title{
INTERNAL DISTRIBUTION
}

\author{
1. A. Choudhury \\ 2. J. F. Cooke \\ 3. F. V. Damiano \\ 4. G. E. Jellison, Jr. \\ 5. D. H. Lowrides \\ 6. T. L. Payne \\ 7. C. T. Rice \\ 8. J. R. Roberto \\ 9. K. M. Wilson, Technology Transfer and Economic Development \\ 10. Laboratory Records - RC
}

\section{EXTERNAL DISTRIBUTION}

11. P. A. Carpenter, Department of Energy, Oak Ridge Operations Office, P.O. Box 2008, Oak Ridge, Tennessee 37831-6269

12. P. M. Dehmer, Acting Director for Materials Sciences and Engineering Division of the Office of Basic Energy Sciences, SC-10/GTN, DOE, Department of Energy, 1000 Independence Ave. SW, Washington, DC 20585-1290

13. C. O. Griffiths, Hinds Instruments, Inc., 3175 NW Alocleck Drive, Hillsboro, Oregon, 97124-7135 